\title{
Research on Reasonable Permeable Grouting Pressure Based on Slurry-coal Body Coupling Effect
}

\author{
Fengfeng Yang ${ }^{1, a}$, , Jufeng Zhang ${ }^{1,2, b}$, Chao Zheng ${ }^{1, ~ c}$, Shuang $\mathrm{Li}^{1}$, and Dongfang Gao ${ }^{1}$ \\ ${ }^{1}$ College of Energy Engineering, Long Dong University, Qingyang 745000, China \\ ${ }^{2}$ School of Resource, Environment and Safety Engineering, Hunan University of Science and \\ Technology, Xiangtan 411201, China

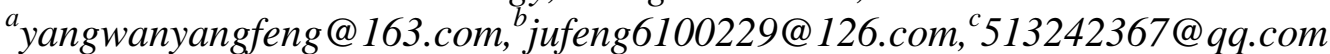

Keywords: percolation grouting; gas-bearing coal; grouting pressure; numerical simulation; coupling effect

\begin{abstract}
When measuring gas pressure in high-pressure gas-containing coal bodies, the quality of sealing pores plays a decisive role in the determination results, and the selection of reasonable grouting pressure during sealing is particularly important. Aiming at the deficiencies in the current grouting pressure research, based on the range of plastic zone, a slurry permeability diffusion model under multi-field coupling was established. According to the theoretical model created, the law of slurry diffusion under different grouting pressures was analyzed, and the reasonable grouting pressure was verified by gas pressure measurement results. The field test results show that the 5MPa grouting pressure simulated by this model is reasonable and can be consistent with the in-situ grouting effect.
\end{abstract}

\section{Introduction}

There are a large number of pores and fissures in the coal body, which is a complex porous medium structure. The particles of this porous medium are not flexible. When grouting into coal and rock, it is difficult for the slurry to penetrate the pore-slit network to compress its skeleton. Hat is to say, the dispersion of the slurry is performed mainly around the porous medium in a" flow-through" manner, supplemented by the pores of the compacted particles ${ }^{[1]}$. In other words, the path of the slurry diffusion is to diffuse in a curved pattern around the porous medium particles, not simply a straight line diffusion. In the case of permeation grouting and plugging for pressure measurement in high-pressure gas-bearing coal bodies, the quality of the sealing quality is particularly important, and the quality of the sealing hole is inextricably linked to the selection of reasonable grouting parameters. Therefore, determining the reasonable grouting parameters based on the fracture conditions of the surrounding rock and the permeability characteristics of the slurry is the premise of ensuring strict sealing of the hole. The penetration of slurry in pores of coal is caused by the coupling of the flow field of the slurry and the stress field where the coal is located. The presence of pore-fissures causes the pressure of the slurry to attenuate in the direction of the slurry diffusion ${ }^{[2-3]}$. Theoretically speaking, to increase the range of slurry diffusion, grouting pressure will inevitably increase with time. When the grouting pressure exceeds the critical pressure 
of the crack deformation, the Cracks will undergo displacement deformation perpendicular to the development direction of the fracture, and a crack phenomenon will occur.

When measuring gas pressure in coal seams containing high-pressure gas, it is still a difficult problem to obtain accurate results for the mines that do not have the condition to drill through-hole drills, especially the frequent mining effects and the complicated conditions such as soft broken coal lanes have brought certain difficulties to the accurate determination of gas pressure ${ }^{[4]}$. The cracks developed around the loosening zone formed during the excavation process of the coal roadway, and in this case, he use of bedding borehole pressure sealing, put forward higher requirements for sealing technology. (1)It is generally believed that the grouting pressure above 8.0MPa can tightly seal the fracture around the borehole ${ }^{[5]}$,but for the coal seam,8.0 MPa may crack the surrounding coal body. If the pressure exceeds compressive strength of the coal body, there may be accidents such as sheet and other accidents. At present, the study of gas pressure measurement and reasonable grouting pressure in gas extraction process is still not deep enough. Yang Hongmin ${ }^{[6-7]}$ and others used a combination of theoretical analysis, numerical simulation and field practice to arrive at a reasonable grouting pressure of $4 \mathrm{MPa}$ when measuring the gas pressure in the borehole of Pingdingshan No. 13Mine.In most other cases, only grouting pressure ${ }^{[8-9]}$ was selected based on engineering experience. There is no scientific theoretical support and there is no engineering validation. In view of this, an in-depth study of the reasonable grouting pressure during the process of gas pressure measurement during the process of measuring the gas pressure has an important guiding role in the field sealing and grouting.

\section{Numerical Simulation of Plastic Stress Zone surrounding Rock Drilling}

During the process of bedding through construction, the drill pipe is continuously circulated and perturbed, and the plastic zone, elastic zone, and original rock stress zone are formed around the borehole. At the same time, along with the redistribution of stress around the coal body around the hole, a stress concentration zone appears around the hole when the strength of the coal body is less than the stress of the coal around the hole, It will cause plastic deformation and damage of the coal around the borehole. The area in the plastic zone where the stress is less than the original stress is the rupture zone in In this area, the stress and strength are significantly reduced, and the expansion of pores and fissures is increased. The larger the plastic zone of the borehole is, the larger the corresponding crack area, which is not conducive to the accurate measurement of gas pressure.

In order to simulate the size of the borehole plastic zone, according to the elasto-plastic theory, the length of the bedding hole is much larger than its cross-sectional area, so the stress problem of the borehole wall can be reduced to plane strain. The following basic assumptions are: (1) The borehole is approximately circular, and the original rock stress in the coal roadway is reduced to a hydrostatic stress field, and the surrounding pressure is uniform and isotropic; (2) The stress distribution around the bedding hole is the same as the stress distribution in the elastic zone, while the coal failure in the plastic zone meets the Mohr-Coulomb yield criterion; (3) The stress of coal and rock mass in the crush zone is lower than that of the original rock.

Based on the above assumptions, the radius of the plastic zone can be obtained as:

$$
R=a\left[\frac{(p+c \cot \varphi)(1-\sin \varphi)}{p_{i}+c \cot \varphi}\right]^{\frac{1-\sin \varphi}{2 \sin \varphi}}
$$

From the formula: the greater the stress $P$ of the original rock, the larger the plastic zone around the borehole; and the smaller the cohesion c and internal friction angle $\varphi$ (That is, the lower the strength of the coal rock body); The larger the pressure drill hole radius $a$, the larger the radius $R$ of the plastic zone. Therefore, a two-dimensional planar model of bedding pressure drilling was 
established to analyze the changes of its surrounding plastic zone. When the depth of burial is $600 \mathrm{~m}$, the initial ground stress is $15 \mathrm{MPa}$.The simulation results after using the comsol software are shown in Figure 1.

The plastic failure zone around the borehole in Fig. 1 has a destruction range of about $65 \mathrm{~mm}$. The cracks in surrounding rock in the plastic zone provide a channel for gas flow and leakage, so the plastic zone of the borehole must be tightly sealed.

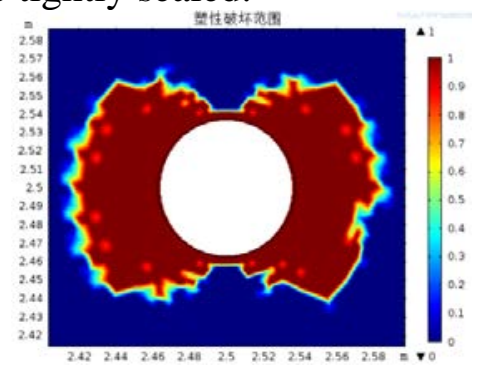

Fig. 1 Plastic failure zone around borehole

\section{Numerical Simulation of the Seepage Characteristics of Slurry at Different Grouting Pressures}

\subsection{Basic assumptions}

Due to the complexity of site conditions, basic assumptions are made in the construction of mathematical models: (1) The percolation motion of the slurry obeys Darcy's law; (2) Coal seam is homogeneous and isotropic; (3) The slurry is an incompressible fluid.

\subsection{The establishment of a mathematical model}

During the grouting process, the moisture air in the fracture under the effect of slurry seepage is exchanged with the slurry, so according to the relevant literature, a mathematical model can be established from the point of conservation of mass ${ }^{[10]}$.In the seepage field of the slurry, any control body $U$ is used. The porosity of the coal is $\varphi$ taken $\mathrm{d} \sigma$ as one element on the outer surface $\sigma$, the outer normal line is $\mathrm{n}$, the seepage velocity of the fluid in the unit is $v$, The mass flowing in unit time is $\rho v \bullet n \mathrm{~d} \sigma$, the total mass is $\oiint_{U} \rho v \bullet n \mathrm{~d} \sigma$ taken $\mathrm{d} U$ as one element of $U$,For unsteady fluid, the density will change with time, which leads to the increase of internal mass $U$ is $\int_{U} \frac{\partial(\rho \varphi)}{\partial t} d U$.Assuming the active (sink) distribution in $U$, then the fluid mass produced by the entire control body $U$ per unit time is $\int_{U} \rho q \mathrm{~d} U$, then the mass conservation equation can be expressed as:

$$
\int_{U} \frac{\partial(\rho \varphi)}{\partial t} d U=\int_{U} \rho q d U-\oiint_{\sigma} \rho v \bullet n d \sigma
$$

Equation (2) is the continuity equation under the integral form. Use the Gaussian formula to convert (2) to:

$$
\int_{U} \nabla \cdot(\rho V) \mathrm{d} U=\oiint_{\sigma} \rho v \bullet n \mathrm{~d} \sigma
$$

Substituting (3) into (2): $\int_{U}\left[\frac{\partial(\rho \varphi)}{\partial t}+\nabla \bullet(\rho v)-q \rho\right] \mathrm{d} U=0$ 
Because the control body $U$ is arbitrary, so long as the integrand is continuous, the continuity equation of the seepage flow of the slurry can be obtained as:

$$
\frac{\partial(\rho \varphi)}{\partial t}+\nabla \cdot(\rho v)=q \rho
$$

For passive unsteady flow, the continuity equation can be expressed as:

$$
\frac{\partial(\rho \varphi)}{\partial t}+\nabla \bullet(\rho v)=0
$$

For grouting projects, the law of slurry flow can be expressed using Darcy's law:

$$
v=-\frac{k}{\mu}(\nabla P+\rho g \nabla D)
$$

From the integration equation (4), (6)

$$
\frac{\partial(\rho \varphi)}{\partial t}+\nabla \cdot \rho\left[-\frac{k}{\mu}(\nabla P+\rho g \nabla D)\right]=q
$$

The governing equation of grouting seepage movement can be expressed as:

$$
S \frac{\partial P}{\partial t}+\nabla\left[-\frac{k}{\mu}(\nabla P+\rho g \nabla D)\right]=\rho q
$$

In the formula: $v$ is the seepage velocity, $\mathrm{m} / \mathrm{s}$; $\mathrm{k}$ is the coal body permeability, $\mathrm{m}^{2} ; \mu$ is the dynamic viscosity, $\mathrm{kg} /(\mathrm{m} \cdot \mathrm{s}) ; P$ is the fluid pressure, $\mathrm{kg} /\left(\mathrm{m} \cdot \mathrm{s}^{2}\right), \rho$ is the slurry density, $\mathrm{kg} / \mathrm{m}^{3} ; \varphi$ is porosity; $q$ is the intensity of source sink; $t$ is the time variable; $s$ is the storage coefficient.

\subsection{Numerical model and boundary conditions}

(1)Engineering conditions: the diameter of pressure measuring drilling is $0.075 \mathrm{~m}$, the drilling and sealing sections are all in the coal seam. (2)The initial conditions and boundary conditions: the borehole wall is the slurry inflow boundary, and there is no slurry flow boundary exists at other boundaries. Initial conditions:

$$
\left.P\right|_{t=0}=P_{0}
$$

$P_{0}$ is the initial pressure at the grouting boundary of the hole.

The pressure gradient of slurry at infinity is 0 . the model boundary condition is:

$$
\left\{\begin{array}{l}
\left.P\right|_{r=0}=P_{0} \\
\left.\frac{\partial P}{\partial r}\right|_{r=l}=0
\end{array}\right.
$$

\subsection{The simulation results}

Using comsol software for simulation, the relationship between the grouting pressure and the slurry diffusion distance generated after the data was extracted in the data set is shown in Figure 2.

It can be seen from Fig. 2 that before the grouting pressure is $5 \mathrm{MPa}$, the slurry diffusion radius increases with the increase of grouting pressure; and the grouting pressure after $5 \mathrm{MPa}$, the diffusion radius of the grouting shows no obvious trend with increasing grouting pressure. At this time, increasing the grouting pressure to increase the slurry radius is not desirable. Therefore, the 
critical value of grouting pressure for coal slurry penetration in coal seams is $5 \mathrm{MPa}$. In addition, when the grouting pressure is $5 \mathrm{MPa}$, the corresponding diffusion radius of the slurry is $108 \mathrm{~cm}$, and the maximum damage area formed after drilling is $65 \mathrm{~mm}$. The spread radius of the slurry is much larger than the damage range of the plastic zone, which can ensure the tight sealing of the fracture around the borehole. In short, the grouting pressure of $5 \mathrm{MPa}$ is theoretically reasonable.

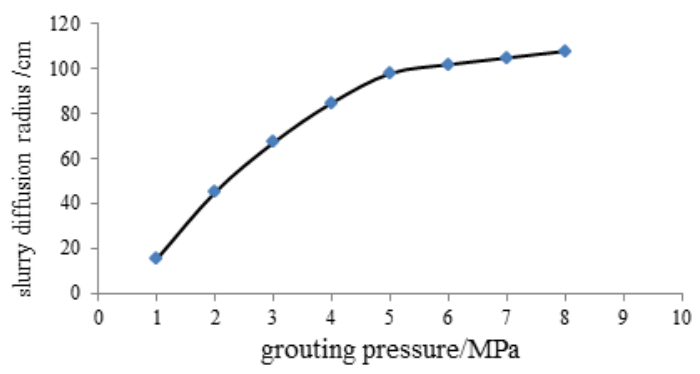

Fig.2 Curve of the diffusion radius of slurry under different grouting pressures

\section{Field test}

\subsection{Overview of test sites}

The production capacity of hou village coal mine is $1.2 \mathrm{million} \mathrm{t} / \mathrm{a}$, and the recoverable coal seam in the well field is No.3,9 and 15 coal seams, with an average total thickness of $10.36 \mathrm{~m}$,and the coal coefficient of the recoverable coal seam is $7.72 \%$. The gas content of coal seam is $6.8 \sim 16.79 \mathrm{~m}^{3} / \mathrm{t}$, and the maximum gas pressure of coal seam is 1.12MPa.Mine absolute gas emission is $67.83 \mathrm{~m}^{3} / \mathrm{min}$, the relative gas emission is $27.12 \mathrm{~m}^{3} / \mathrm{t}$, were chosen in 3601 working face, to a length of $1062 \mathrm{~m}$,the tendency for the length of $160 \mathrm{~m}$,average thickness of coal seam $6.02 \mathrm{~m}$. The roof is mudstone or silty sandstone, and the floor is mudstone or silty sandstone. The simulation uses the average depth of $560 \mathrm{~m}$.

\subsection{Test results}

Hou village coal mine used polyurethane hole sealing methods for coal seam gas pressure determination, because of the limitations on polyurethane sealing hole itself, leading to low pressure results. Under the pressure of 5MPa grouting, the gas pressure was measured by the" two plug and one injection" belt pressure sealing technology at the same site in the 3601 working face. Two pressure measuring holes are arranged at intake airway 230m (No.1 hole) and 260m (No.2 hole) at the 3601 working face of six mining area. The depth of the hole is $30 \mathrm{~m}$, the depth of the sealing is $25.5 \mathrm{~m}$ and $26 \mathrm{~m}$ respectively. The results of gas pressure measured are shown in figures 3 and 4 .

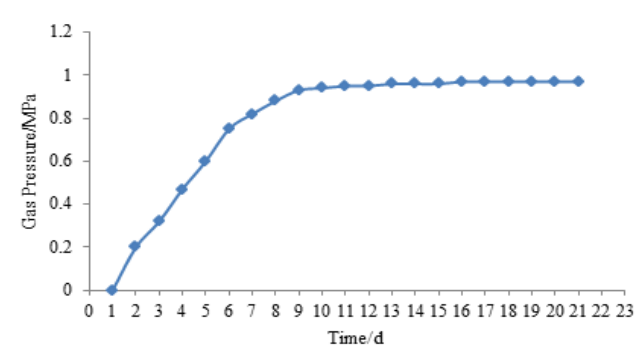

Fig.3 1 hole gas pressure recovery curve

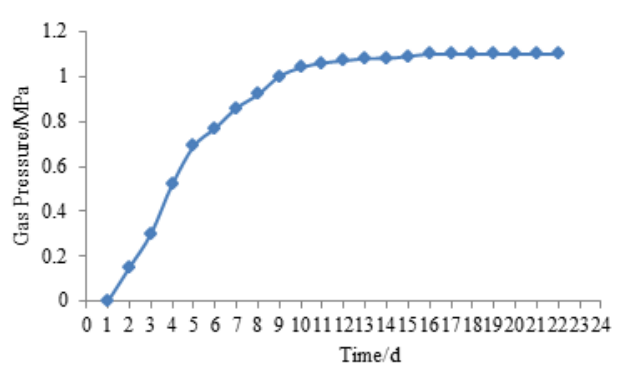

Fig.4 2 hole gas pressure recovery curve 
According to the on-site sealing pressure measurement method and results, It shows that under the same conditions, the pressure of grouting plays a key role in the sealing quality and the measured gas pressure is a good proof of the rationality of the grouting pressure.

\section{Conclusion}

The diffusion path in the process of slurry infiltration was analyzed. Based on the numerical simulation results of the drilling plastic zone and the plastic zone of the roadway, according to the coupling effect between coal stress field and slurry flow field, a mathematical model of the slurry flow and diffusion radius was established, the law of slurry diffusion under different grouting pressures was analyzed, and a reasonable grouting pressure was 5MPa.The site test was performed using the" two-injection and one-injection" capsule sealing instrument. The accurate gas pressure measurement results proved that the 5MPa grouting pressure was reasonable.

\section{Acknowledgments}

This work was financially supported by 2018 Gansu Provincial Safety Science and Technology Project[GAJ00011] and Longdong University Youth Science and Technology Innovation Project[XYZK1610] fund.

\section{References}

[1] Zhang Lianzhen, Zhang Qingsong, Liu Rentai, et al. Research on Grouting Mechanism in Fractured Rock Considering Slurry-Rock Stress Coupling Effects[J]. Chinese Journal of Geotechnical Engineering,1-9.

[2] Zhang Qingsong, Wang Hongbo, Liu Rentai, et al. Infiltration grouting mechanism of porous media considering diffusion paths of grout[J]. Chinese Journal of Geotechnical Engineering,2018,40(05):918-924.

[3] Ruan Wenjun. Spreading model of grouting in rock mass fissures basend on time-dependent behavior of viscosity of cement-basend grouts[J]. Chinese Journal of Rock Mechanics and Engineering,2005, (15):2709-2714.

[4] Yue Gaowei, Yuan Junwei, Feng Fan, et al. Numerical Simulation of Surrounding Rock Stability in the Excavation Roadway with Trapezoidal Section[J]. Safety in Coal Mines,2012, 43 (11):45-47.

[5] Wang Hao, Jiang Chenglin, Zhang Jianjun, et al. Novel Technology Studies on Drilling Grouting in Methane Pressure Measurement of Coal Seam[J]. China Safety Science, Journal,2011, 21(6):78-83.

[6] Yang Hongmin, Yang Fengfeng, Chen Xiangjun, et al. Automatic Pressure Measuring Technology with "Two Sealing and One Grouting" Capsule Fast Sealing Technology in Cross-layer Borehole[J]. Safety in Coal Mines,2015,11(5):13-17.

[7] Yang Hongmin, Yang Fengfeng, An Fenghua, et al. Study on reasonable grouting sealing pressure for Hole Sealing in the Determination of Coal Seam Gas Pressure [J]. Journal of Safety Science and Technology, 2015,46(5):78-80.

[8] Li Shengang, Zhao Wen, Du Jiahong, et al. Research Situation and Prospect on Osmotic Grouting Test[J]. Subgrade Engineering,2010, (3):34-36.

[9] Sun Bintan, Ling Xianzhang, Ling Chen, et al. Numerical simulation for diffusion and pressure distribution of permeation grouting[J]. Journal of Hydraulic Engineering,2007, 37(11):1402-1406.

[10] Li Shucai, Zheng Zhuo, Liu Rentai, et al. Analysis of diffusion of grout in porous media considering infiltration effects [J]. Chinese Journal of Rock Mechanics and Engineering.34(12), 2401-2409. 